Altai State University

Acta 3 iwlogica Gibirica

Journal of Biology

Founded in 2015
WWW.asu.ru

ISSN 2412-1908

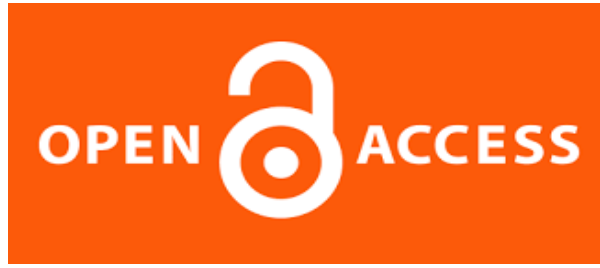

Acta Biologica Sibirica, 2018, 4(2), 25-31

\title{
An annotated checklist of Dolichopodidae (Diptera) of Chukotka (Russia) with new records
}

\author{
I.Ya. Grichanov \\ All-Russian Institute of Plant Protection, Podbelskogo 3, St. Petersburg, Pushkin, 196608 Russia \\ E-mail: grichanov@mail.ru
}

A checklist of Chukchi species is compiled. The present research gives new records, including 26 species found for the first time in Chukotka, three species recorded for the first time in the Palaeartic Region (Argyra albiventris Loew, 1864, Hydrophorus pilitarsis Malloch, 1919, Rhaphium nigrum (Van Duzee, 1923)) in addition to one species new for Russia (Dolichopus pseudomigrans Ringdahl, 1928). In total, 8 genera and 36 species are listed that apparently makes up less than $50 \%$ of actual Dolichopodidae fauna of the Okrug. Now 17 Chukchi dolichopodids (47\%) are known in the Nearctic Region. Key words: Dolichopodidae; Chukotka Autonomous Okrug; new record

\section{Introduction}

Chukotka Autonomous Okrug or Chukotka is geographically located in the Far East region of Russia. Chukotka is bordered in the north by the Chukchi Sea and the East Siberian Sea, which are part of the Arctic Ocean; in the east by the Bering Strait and the Bering Sea, part of the Pacific Ocean; in the south by Kamchatka Krai and Magadan Oblast; and in the west by the Sakha Republic (Fig. 1). The Chukchi Peninsula projects eastward forming the Bering Strait between Russia and Alaska, and encloses the north side of the Gulf of Anadyr. Chukotka Okrug includes the Wrangel Island in the Arctic Ocean and some other smaller islands.

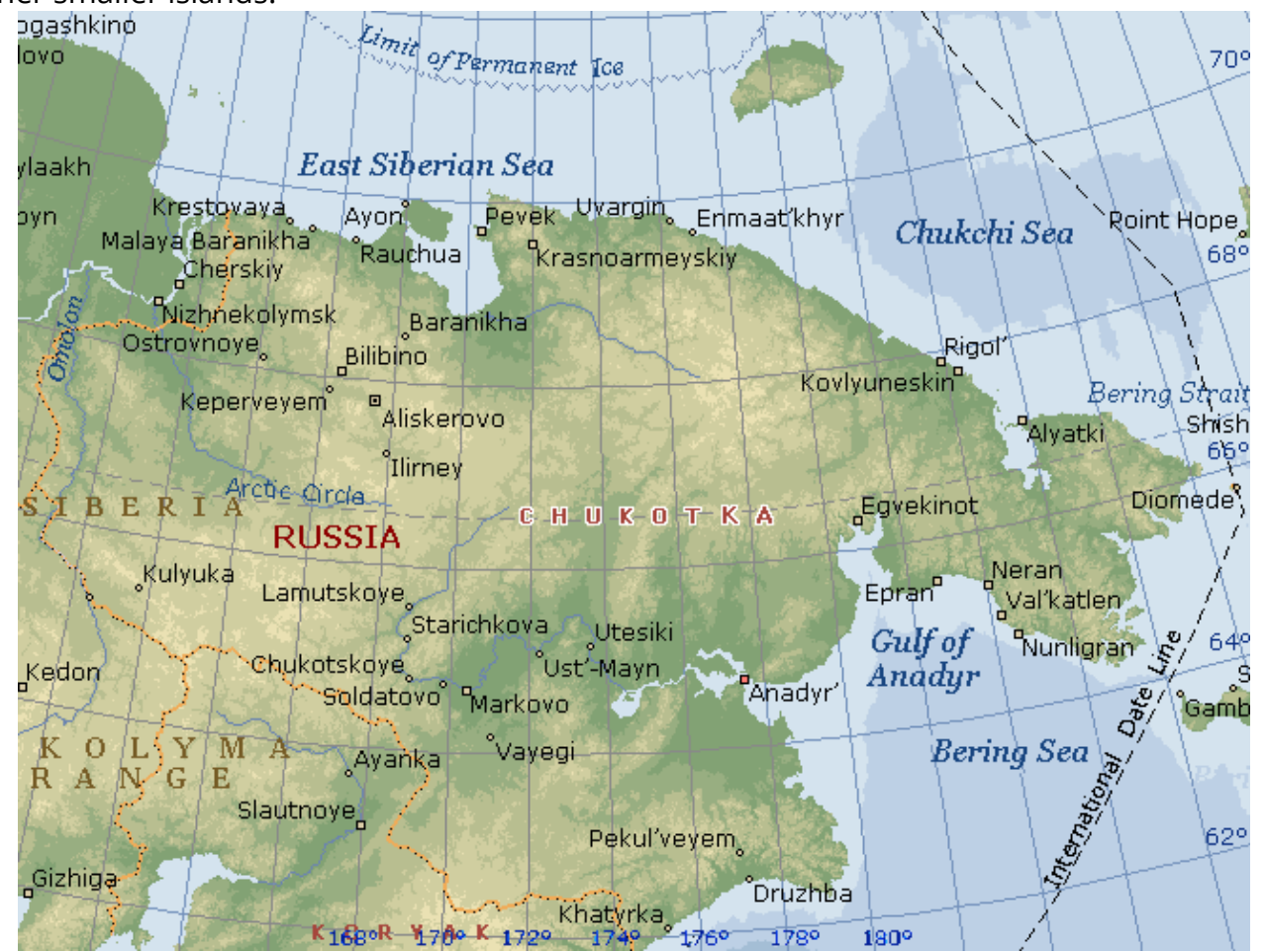

Fig. 1. Map of the Chukotka Autonomous Okrug. 
Grichanov, I.Ya. An annotated checklist of Dolichopodidae of Chukotka.... Acta Biologica Sibirica, 2018, 4(2), 25-31

Ecologically, Chukotka can be divided into three distinct areas: the northern Arctic desert, the central tundra, and the taiga in the south. About half of its area is above the Arctic Circle. This area is very mountainous, containing the Chukotsky Mountains and the Anadyr Range.

In the case of long-legged flies, the territory of the Chukotka Autonomous Okrug is one with the poorly studied fauna of long-legged flies in Russia (Negrobov et al., 2013; Grichanov, 2017) despite its significant location near the Nearctic zoogeographical region. The oldest material collected from the territory ("Chukotka Peninsula", "Chaunskaya Guba, Ichun River mouth"; females only) and found in the Zoological Institute of the Russian Academy of Sciences, St. Petersburg (ZIN) was cited by Negrobov, Maslova (1995). Two females collected in 1900 by I.N. Akifiev and in 1939 by G. Semenov were included into the type series for the Far-Eastern Chrysotus nudisetus. The holarctic-hypoarctic Hydrophorus alpinus Wahlberg, 1844 was recorded from the localities "Chukotka Peninsula, cape Serdce Kamenj" and "Chaunskaya Guba, Ichun River mouth" (Maslova et al., 2011). Two specimens collected in 1911 by L.M. Starokadomskii and in 1940 by G. Semenov were mentioned by the authors. Hydrophorus arcticus Negrobov, 1977 was described by a series collected in 1913 by Starokadomskii at the Kalyutschinskaya Guba (Negrobov, 1977).

The professional collection of Diptera from Chukotka was started by the famous Soviet dipterologist K.B. Gorodkov (ZIN), since late 1950s. Four species were described and reported from this territory after his samples (Grichanov, 2012a, 2012b; Negrobov, Grichanov, 2013; Negrobov et al., 2016a). Chrysotus chukotkensis Grichanov, 2012 was described from environs of Egvekinot (1963 expedition). Mainly boreal Dolichopus cinctipes Wahlberg, 1850 was recorded from the Bolshaya River upper reaches (1959 expedition) and Bilibino (1971 expedition). Circum-arctic Dolichopus humilis Van Duzee, 1921 was recorded from Egvekinot and Pevek environs (1963 expedition). Sympycnus gorodkovi Negrobov, Barkalov, Selivanova et Grichanov, 2016, was found by Gorodkov at the Bolshaya River upper reaches (1959 expedition). Nevertheless, the most part of the Gorodkov's samples (in the ZIN collection) was only recently sorted to a family level, being treated in this paper. In addition, Dr. A.V. Barkalov (the Institute of Systematics and Ecology of Animals of Siberian Branch of Russian Academy of Sciences, Novosibirsk) has found a female Rhamphium beringiense Negrobov et Vockeroth, 1979, in a rather cold south-eastern part of the Wrangel Island in 1975 (Negrobov et al., 2012).

In 2006 and 2015, O.A. Khruleva (the A.N. Severtsov Institute of Ecology and Evolution of the Russian Academy of Sciences, Moscow) has collected a lot of new dolichopodid material from the Wrangel Island, and I.Ya. Grichanov has identified five species from her samples including Chrysotus komovi Negrobov, Barkalov et Selivanova, 2014, and Dolichopus terminasianae Negrobov, Selivanova et Maslova, 2011 found for the first time in the Chukotka Autonomous Okrug (Grichanov, Khruleva, 2018).

During the recent 2009, 2012, 2016 and 2017 surveys conducted on the Chukotka Peninsula, about 90 specimens of Dolichopodidae have been collected by Dr. P.S. Tomkovich and treated in this paper. They will be deposited in the Zoological Museum of Moscow State University, Moscow, Russia (ZMUM).

\section{A checklist of species and new records of Dolichopodidae}

Species listed below are arranged alphabetically by genus. For each species we provide references (if available) for the published material; unpublished material studied; general distribution of species (after Grichanov, 2017). The type localities are provided and the country lists are arranged alphabetically. Remarks are provided where deemed necessary. Specimens are mounted on pins and placed in the museum drawers.

\section{Argyra Macquart, 1834}

Argyra albiventris Loew, 1864

MATERIAL. Bilibino, marsh, 5-6 vii 1971, Gorodkov, $13 \overbrace{}^{\lambda}$.

Type locality: USA: Alaska, Sitka. Nearctic: from Alaska southward to Oregon and Utah. New for the Palaeartic Region.

Campsicnemus Haliday, 1851

Campsicnemus pumilio (Zetterstedt, 1843)

MATERIAL. Meynypilgyno, $62.6^{\circ} \mathrm{N}, 177.02^{\circ} \mathrm{E}, 28$ vi 2012, 27 vii 2016, 2 viii 2016, P. Tomkovich, 5 q (ZMUM).

DISTRIBUTION. Type locality: Sweden: Sueciam in Scania ad Scandhammar et Silfakra. Palaearctic: Austria, Belgium, Czech, Denmark, Estonia, Finland, France, Germany, N Kazakhstan, Kyrgyzstan, Netherlands, Poland, Romania, Russia (Karelia, Krasnodar, Krasnoyarsk, Leningrad, Moscow, Novgorod, Ryazan, Ulyanovsk, Yakutia, Kamchatka), Sweden, UK.

\section{Chrysotus Meigen, 1824}

Chrysotus chukotkensis Grichanov, 2012

REFERENCES. Grichanov, 2012a: 333 (Egvekinot).

DISTRIBUTION. Type locality: Chukotka, 5 km N Egvekinot. Palaearctic: Russia (Chukotka).

Chrysotus gramineus (Fallén, 1823)

REFERENCES. Negrobov, Chalaya, 1991: 67. 
Grichanov, I.Ya. An annotated checklist of Dolichopodidae of Chukotka.... Acta Biologica Sibirica, 2018, 4(2), 25-31

DISTRIBUTION. Type locality: not given [Sweden]. Palaearctic: Abkhazia, Andorra, Armenia, Austria, Azerbaijan, Belarus, Belgium, Bosnia and Herzegovina, Bulgaria, China; Czech Republic, Denmark, Estonia; Finland, France, Georgia, Germany, Greece, Hungary, Ireland, Italy, Korea, Kyrgyzstan, Latvia, Lithuania, Moldova, Netherlands, Norway, Poland, Romania, Russia (Adygea, Alania, Altai Rep., Arkhangelsk, Bashkortostan, Blagoveshchensk, Buryatia, Chelyabinsk, Chita, Chukotka, Crimea, Irkutsk, Kabardino-Balkaria, Karachai-Cherkessia, Karelia, Khabarovsk, Kostroma, Krasnodar, Krasnoyarsk, Kursk, Leningrad, Lipetsk, Mordovia, Moscow, Murmansk, Novgorod, Orel, Orenburg, Pskov, Ryazan, Sakhalin, Tatarstan, Tomsk, ?Ural, Vologda, Voronezh, Yakutia, Yaroslavl), Slovakia, ?Slovenia, Spain, Sweden, Switzerland, Tajikistan, Turkey (Hakkari, Erzurum, Kars), Turkmenistan, UK, Ukraine (Cherkasy, Kharkiv, Kherson).

REMARKS. Negrobov, Chalaya (1991) mentioned one specimen (with no sex indicated) collected in 1939 by G. Semenov at the locality "Chaunskaya Guba, Ichun River mouth". Later Negrobov together with his disciples described several new species closely related to Chrysotus gramineus from the Far East (e.g. Negrobov, Maslova, 1995; Maslova, Negrobov, 2015; Negrobov et al., 2014, 2015, 2016b). Therefore, a record of C. gramineus from Chukotka must be confirmed as it can belong to another species.

\section{Chrysotus komovi Negrobov, Barkalov et Selivanova, 2014}

REFERENCES. Grichanov, Khruleva, 2018: 25 (Wrangel Island).

MATERIAL. Pevek, Dryas tundra, 28-29 vi 1963, Gorodkov (ZIN), 6ð̋; Komsomolskii, Chaunskii District, 4 vii 1963 , Gorodkov (ZIN), $1 \hat{\jmath}$.

DISTRIBUTION. Type locality: Russia: Krasnoyarskij Kraj, Taimyr Reserve, Ary-Mas field station, left bank of river Novaya. Palaearctic: Russia (Chukotka, Taimyria).

Chrysotus nudisetus Negrobov et Maslova, 1995

REFERENCES. Negrobov, Maslova, 1995: 460 (“Chukotka Peninsula”, “Chaunskaya Guba, Ichun River mouth”; females only).

DISTRIBUTION. Type locality: Russia: Sakhalin, Anivskii distr. Palaearctic: Japan, Russia (Chukotka, Khabarovsk, Magadan, Sakhalin, Vladivostok, Yakutia).

\section{Dolichopus annulipes Zetterstedt, 1838}

MATERIAL. Upper reaches of Bolshaya River, $63^{\circ} 01^{\prime} \mathrm{N}, 171^{\circ} 50^{\prime} \mathrm{E}, 23$ vii 1959, Gorodkov (ZIN), $1 \mathrm{O}^{\text {} .}$.

DISTRIBUTION. Type locality: Sweden: Lapponia Umensi; Lycksele; Lapponia Dalekarlia. Palaearctic: Denmark, Estonia, Finland, Latvia, Norway, Russia (“Caucasus”, Arkhangelsk, Karelia, Komi, Leningrad, Murmansk, Buryatia, KhantiaMansia, ?Magadan, ?Vladivostok, Yamalia), Sweden; Nearctic: Alaska, Yukon Territory, British Columbia, Ontario to Newfoundland (Labrador), southward to New York.

\section{Dolichopus armillatus Wahlberg, 1850}

MATERIAL. Bilibino, marsh, 5 vii 1971, Gorodkov, 14ર̊ํ (ZIN); lultin, 20 vii 1963, Gorodkov, $1 \delta^{\lambda}$ (ZIN); Anadyr distr., Meynypilgyno env., $63.567^{\circ} \mathrm{N}, 177.033^{\circ} \mathrm{E}, 6-10$ vii 2009, 28 vi 2016, coll. P.S. Tomkovich, $2 \delta^{\jmath}, 1$ (ZMUM).

DISTRIBUTION. Type locality: Quickjock, Lapponiae Lulensis, Mounioniska, Peljatschware, Koutokeino, Finmarkiae, Syvajarvi infra alpem Stuor Oive [Sweden \& Finland]. Palaearctic: Czech, Finland, Norway, Russia (Karelia, Murmansk, N Ural, Magadan), Sweden.

\section{Dolichopus cinctipes Wahlberg, 1850}

REFERENCES. Grichanov, 2012 b: 317 (Bolshaya River upper stream; Bilibino).

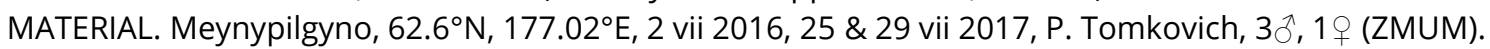

DISTRIBUTION. Type locality: Sweden: Rubo Chamaemaemoro, Gaskaivo, Quickjock, Njunnas. Palaearctic: Finland, Norway, Russia (Arkhangelsk, Blagoveshchensk, Buryatia, Chukotka, Khabarovsk, Komi, Magadan, Murmansk, North Ural, Vladivostok), Sweden, Turkey (Erzurum, Kars).

\section{Dolichopus discifer Stannius, 1831}

MATERIAL. Meynypilgyno, 62³7.19’N, 17650.72'E, lake, 2 vii 2016, P. Tomkovich, $10^{\Uparrow}$ (ZMUM).

DISTRIBUTION. Type locality: Germany. Palaearctic: Austria, Belgium, Belarus (Minsk), Bulgaria, Czech, Denmark, Estonia, Finland, France, Georgia, Germany, Hungary, Ireland, Italy, N Kazakhstan, Latvia, Lithuania, Netherlands, Norway, Poland, Romania, Russia (Altai Rep., Baikal, Karelia, Khabarovsk, Leningrad, Mordovia, Moscow, Murmansk, Novgorod, Sakhalin,Vladivostok, Vologda, Yakutia), Slovakia, Sweden, Switzerland, UK, Ukraine (Kharkiv); Nearctic: Canada and USA: Alaska, British Columbia to Quebec and Nova Scotia, southward to Colorado and New York.

\section{Dolichopus fraterculus Zetterstedt, 1843}

MATERIAL. Upper reaches of Bolshaya River, $63^{\circ} 01^{\prime} \mathrm{N}, 171^{\circ} 50^{\prime} \mathrm{E}, 24$ vii 1959, Gorodkov, $1 \delta^{\lambda}$ (ZIN); Anadyr aeroport, N bank at village, 31 vii 1963, Gorodkov, 10 (ZIN).

DISTRIBUTION. Type locality: Sweden: Jemtlandia meridionali, Åsarne. Palaearctic: Finland, Lithuania, Norway, Sweden, Russia (Altai Rep., Karelia, Kuril Is., Magadan, Murmansk, Yamalia, Yakutia); Nearctic: Alaska, Northwest Terr. 
Grichanov, I.Ya. An annotated checklist of Dolichopodidae of Chukotka.... Acta Biologica Sibirica, 2018, 4(2), 25-31

\section{Dolichopus humilis Van Duzee, 1921}

REFERENCES. Negrobov, Grichanov, 2013: 499 (Egvekinot and Pevek environs); Grichanov, Khruleva, 2018 : 26 (Wrangel Island).

DISTRIBUTION. Type locality: USA: Alaska-Yukon Territory boundary. Palaearctic: Russia (Chukotka, Taimyria, N Yakutia, Yamalia); Nearctic: USA (Alaska), Canada, Greenland.

\section{Dolichopus longicornis Stannius, 1831}

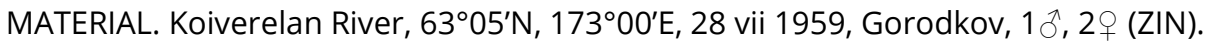

DISTRIBUTION. Type locality: not given [Germany]. Palaearctic: Austria, Belarus, Belgium, China, Czech, Denmark, Estonia, Finland, France, Germany, Hungary, Ireland, Italy, Kazakhstan, Latvia, Lithuania, Luxembourg, Mongolia, Netherlands, Norway, Poland, Romania, Russia (Altai Rep., Arkhangelsk, Blagoveshchensk, Irkutsk, Kamchatka, Karelia, Komi, Krasnodar, Krasnoyarsk, Kuril Is., Leningrad, Lipetsk, Magadan, Moscow, Murmansk, Novgorod, Perm, Pskov, Sakhalin, Sayany, Ural, Vladivostok, Vologda, Voronezh, Yakutia, Yaroslavl), Serbia, Slovakia, Sweden, Switzerland, UK, Ukraine (Kherson, Carpathians); Nearctic: Canada (Yukon), USA (Alaska).

\section{Dolichopus maculipennis Zetterstedt, 1843}

MATERIAL. Upper reaches of Bolshaya River, $63^{\circ} 01^{\prime} \mathrm{N}, 171^{\circ} 50^{\prime} \mathrm{E}, 18$ vii 1959, Gorodkov, $1 \delta^{\wedge}$ (ZIN); Meynypilgyno, $62.6^{\circ} \mathrm{N}, 177.02^{\circ} \mathrm{E}, 6$ vii 2012, 21 vi 2016, 28 vi 2016, P. Tomkovich, 30, 3 + (ZMUM).

DISTRIBUTION. Type locality: Scandinaviae septentrionalis, Jamtlandia boreali ad Skalstugan; Nordlandia Norvegicae, ad Bjorkvik, Hacksten, Schiervo, Giebostad [Sweden, Norway]. Palaearctic: Denmark, Estonia, Finland, Germany, Norway, Russia (Altai Rep., Kamchatka, Leningrad, Magadan, Murmansk, Yakutia), Sweden, UK; Nearctic: Alaska.

\section{Dolichopus plumipes (Scopoli, 1763)}

REFERENCES. Grichanov, 2014: 342 (no material provided). (ZMUM).

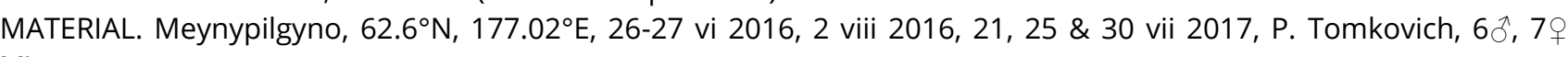

DISTRIBUTION. Type locality: Slovenia: “Carnioliae indigena”. Palaearctic Asia (Afghanistan, China, Kazakhstan, Mongolia, Turkey, Uzbekistan, Russia (Baikal, Chukotka, Irkutsk, Kamchatka incl. Bering Is., Khabarovsk, Khantia-Mansia, Koryakia, Magadan, Novosibirsk, Tomsk, Tyumen, Vladivostok, Yakutia), Caucasus, Europe; Nearctic, Neotropical and Oriental Regions.

\section{Dolichopus pseudomigrans Ringdahl, 1928}

MATERIAL. Upper reaches of Bolshaya River, $63^{\circ} 01^{\prime} \mathrm{N}, 171^{\circ} 50^{\prime} \mathrm{E}$, Chosenia macrolepis forest, 23 vii 1959, Gorodkov, $1 \delta^{\prime}$ (ZIN).

ADDITIONAL MATERIAL EXAMINED. Russia: Nenetsia, Naryan-Mar, fir light forest, 7 viii 1978, Gorodkov, $1 \hat{\jmath}$ (ZIN).

DISTRIBUTION. Type locality: not given. Palaearctic: Finland, Sweden. New for Russia.

\section{Dolichopus rupestris Haliday, 1833}

MATERIAL. Bilibino, marsh, 5-6 vii 1971, Gorodkov, $11 \delta^{1}$ o (ZIN); Upper reaches of Bolshaya River, $63^{\circ} 01^{\prime} \mathrm{N}, 171^{\circ} 50^{\prime} \mathrm{E}$, 12, 14, 16, 25, 27, 30 vii 1959, Gorodkov, $20{ }^{\wedge}$ ㅇ (ZIN); Pevek, Valkumei, see shore, southern slope, bush tundra, 13 vii 1963,

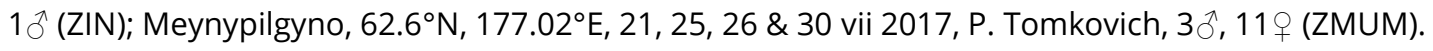

DISTRIBUTION. Type locality: England: Downshire, Tullymore Park and Mountains of Mourne. Palaearctic: Austria, Belgium, China (Xinjiang), Czech, Denmark, Finland, France, Germany, Ireland, Italy, Kazakhstan, Latvia, Lithuania, Netherlands, Norway, Poland, Russia (Altai Rep., Arkhangelsk, Bering Is., Buryatia, Kamchatka, Karelia, Khabarovsk, Kuril Is., Leningrad, Murmansk, Nenetsia, Voronezh, Vladivostok, Yakutia, Ural), Sweden, Switzerland, UK; Nearctic: USA (Alaska), Canada (Yukon Territory).

\section{Dolichopus shantaricus Stackelberg, 1933}

MATERIAL. Apapelkhin, 15 km NOO Pevek, 14 vii 1963, 7 vii 1971, Gorodkov, 5ô, 1 우 (ZIN).

DISTRIBUTION. Type locality: Russia: Shantar-Insel, nordlich von der Amur-Mundung. Palaearctic: Russia (Khabarovsk).

\section{Dolichopus socer Loew, 1871}

MATERIAL. Bilibino, marsh, 5-6 vii 1971, Gorodkov, 32 ${ }^{\top}$ ㅇ (ZIN); Meynypilgyno, $62.6^{\circ} \mathrm{N}, 177.02^{\circ} \mathrm{E}, 2$ vii $2016,25,26$ \& 29 vii 2017, P. Tomkovich, 4ð, 7q (ZMUM).

DISTRIBUTION. Type locality: Russia: “Ochotsk". Palaearctic: Kazakhstan, Mongolia, Russia (Altai Rep., Blagoveshchensk, Buryatia, Kamchatka, Khabarovsk, Krasnoyarsk, Magadan, Tomsk, Yamalia, Yakutia).

REMARKS. Dolichopus socer is almost identical to the Nearctic D. shelfordi Curran, 1934 (not seen) known from Alaska and Manitoba, and the two names are possible synonyms. 
Grichanov, I.Ya. An annotated checklist of Dolichopodidae of Chukotka.... Acta Biologica Sibirica, 2018, 4(2), 25-31

\section{Dolichopus terminasianae Negrobov, Selivanova et Maslova, 2011}

REFERENCES. Grichanov, Khruleva, 2018: 26 (Wrangel Island).

MATERIAL. Bilibino, marsh, 5-6 vii 1971, Gorodkov, 9ð̊o (ZIN); Apapelkhin, 15 km NOO Pevek, 14-15 vii 1963, Gorodkov, $3 \hat{\jmath}$ (ZIN); Pevek, 11 vii 1963, Gorodkov, $1 \hat{\jmath}$ (ZIN); Anadyr aeroport, N bank at village, 1 viii 1963, Gorodkov, $2 \hat{\jmath}$ (ZIN); Krasnoarmeiskii, Chaunskii District, 10 vii 1963, Gorodkov (ZIN), 1 §.

DISTRIBUTION. Type locality: Magadan obl., Aborigen peak, 100 km N Ust'-Omchug, Annachag ridge, Olen' river. Palaearctic: Russia (Chukotka, Magadan, Yakutia).

\section{Hydrophorus alpinus Wahlberg, 1844}

REFERENCES. Negrobov, Chalaja, 1991: 76 (Chaunskaya Guba); Maslova et al., 2011: 93 (Cape Serdtse Kamen; Chaunskaya Guba); Gruzdev et al., 2014: 23 (Wrangel Is.; no material provided); Grichanov, Khruleva, $2018: 26$ (Wrangel Island).

MATERIAL. 55 km N Egvekinot along road, 29 vii 1963, Gorodkov (ZIN), 7ð̋o ; 5 km N Egvekinot, 27 vii 1963, Gorodkov (ZIN), 1 ; Komsomolskii, Chaunskii District, Ichuviem River valley, 5 vii 1963, Gorodkov (ZIN), $6 \bigcirc^{\lambda}$; Apapelkhin, 15 km NOO

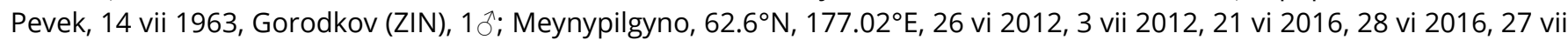
2016, 2 viii 2016, 30 vii 2017, P. Tomkovich, 15ภ, 7q (ZMUM).

DISTRIBUTION. Type locality: Sweden: "Walli et Snjerack prope Quickjock". Palaearctic: Finland, Norway, Russia (Arkhangelsk, Chukotka, Leningrad, Murmansk, Nenetsia, Yakutia, Yamalia), Sweden; Nearctic: USA (Alaska), Canada (Yukon Territory, Northwest Territories, Manitoba, Quebec).

\section{Hydrophorus arcticus Negrobov, 1977}

REFERENCES. Negrobov, 1977: 363 (Kalyuchinskaya Guba).

MATERIAL. Shmidt [=Mys Shmidta], moss-sedge tundra, lake bank, 18 vii 1963, Gorodkov, 1 § (ZIN).

DISTRIBUTION. Type locality: Russia: “Nat.-Kreis Tschuktschen, Kaljutschinskaja guba”. Palaearctic: Russia (Chukotka, Komi, Yamalia, Yakutia).

\section{Hydrophorus geminus Frey, 1915}

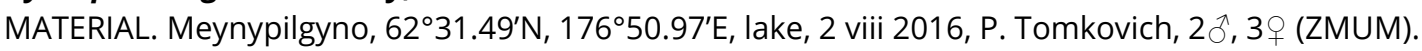

DISTRIBUTION. Type locality: Finland: Ylitomio, am Ufer des Muonio-Flusses, Muonio, Fjelde Olostunturi, Enontekis, Kantalaks. Palaearctic: Finland, Russia (Karelia, Murmansk, West Siberia, Kamchatka), Sweden.

\section{Hydrophorus nebulosus Fallén, 1823}

REFERENCES. Negrobov, 1977: 375 (no material provided).

DISTRIBUTION. Type locality: Sweden: Esperod, Smolandia. Palaearctic: Austria, Belgium, Czech, Denmark, Estonia, Finland, France, Germany, Ireland, Latvia, Netherlands, Norway, Poland, Russia (Buryatia, Chukotka, Karelia, Leningrad, Murmansk, Tyumen), Sweden, Switzerland, UK.

\section{Hydrophorus pilitarsis Malloch, 1919}

MATERIAL. Apapelkhin, 15 km NOO Pevek, 15 vii 1963, 33ึ; 27 vi 1963, Gorodkov, 2 + (ZIN).

DISTRIBUTION. Type locality: USA: Alaska, Teller. Nearctic: USA (Alaska), Canada (Northwest Territories). New to the Palaearctic Region.

REMARKS. The species has almost identical modification of leg with that in the European Hydrophorus rogenhoferi Mik, 1874, but being rather different in male hypopygium morphology (Negrobov, 1977; Hurley, 1985). H. rogenhoferi was once recorded from Siberia (Becker, 1917); however, Negrobov (1977) excluded it from the fauna of Russia.

\section{Hydrophorus praecox (Lehmann, 1822)}

REFERENCES. Negrobov et al., 2000: 85 (no material provided).

DISTRIBUTION. Type locality: Germany: Hamburg. Palaearctic: Abkhazia, Austria, Belgium, Bulgaria, China, Cyprus, Czech Republic, Denmark, Egypt, Estonia, Finland, France, Georgia, Germany, Golan Heights, Greece, Hungary, Iraq, Iran, Ireland, Israel, Kazakhstan, Latvia, Mongolia, Netherlands, Norway, Poland, Portugal (Azores), Romania, Russia (Astrakhan, Chechnya, Chukotka, Crimea, Kabardino-Balkaria, Karelia, Khabarovsk, Krasnodar, Krasnoyarsk, Leningrad, Murmansk, Stavropol, Voronezh), Slovakia, Spain, South Arabia, Sweden, Switzerland, Tunisia, Turkey (Antalya, Bolu), UK, Ukraine (Kherson, Odessa); Oriental: China, India; Australasian: Australia, French Polynesia, New Zealand; Afrotropical: Aldabra, Angola, Botswana, Gambia, Cape Verde Is., Ethiopia, Kenya, Mauritania, Mauritius, Namibia, Nigeria Rodriguez, Senegal, South Africa, St Helena, Tanzania; Neotropical: Chile.

\section{Hydrophorus signifer Coquillett, 1899}

MATERIAL. Shmidt [=Mys Shmidta], moss-sedge tundra, 18 vii 1963, Gorodkov, 1 đ̄ (ZIN).

DISTRIBUTION. Type locality: Russia: Bering Is. Palaearctic: Austria, Belgium, Czech, Finland, Latvia, Netherlands, Norway, Sweden, Russia (Kamchatka, Karelia, Murmansk, Nenetsia, N Ural, Yakutia); Nearctic: USA (Alaska), Canada (Yukon Territory, Northwest Territories, Manitoba, Quebec, Newfoundland). 
Grichanov, I.Ya. An annotated checklist of Dolichopodidae of Chukotka.... Acta Biologica Sibirica, 2018, 4(2), 25-31

\section{Rhaphium beringiense Negrobov et Vockeroth, 1979}

REFERENCES. Negrobov et al., 2012: 76 (19, Wrangel Is.); Grichanov, Khruleva, 2018: 27 (Wrangel Island).

DISTRIBUTION. Type locality: Russia: Magadanskaya oblast, Hazjin river. Palaearctic: Russia (Altai Rep., Chukotka, Kamchatka, Magadan, Taimyria); Nearctic: USA (Alaska).

\section{Rhaphium dichromum Negrobov, 1976}

MATERIAL. 55 km N Egvekinot along road, 29 vii 1963, Gorodkov (ZIN), 13; Komsomolskii, Chaunskii District, 7 vii 1963, Gorodkov (ZIN), 2 . . Magadan).

DISTRIBUTION. Type locality: Russia: Buryat ASSR, Barguzin reservation. Palaearctic: Russia (Altai Rep., Buryatia,

\section{Rhaphium discolor Zetterstedt, 1838}

MATERIAL. Bilibino, marsh, 5 vii 1971, Gorodkov, $2{ }^{\wedge}{ }^{\circ}$ (ZIN); Meynypilgyno, 62.6º $, 177.02^{\circ} \mathrm{E}, 26$ vii 2017, P. Tomkovich, 2 + (ZMUM).

DISTRIBUTION. Type locality: Sweden: Lapponia Umensi; Lycksele; ripa lacus Borgsjoe; Asele; Lapponia meridionalis. Palaearctic: Austria, Belarus, Belgium, Denmark, Estonia, Finland, France, Germany, Ireland, Kyrgyzstan, Latvia, Mongolia, Netherlands, Norway, Poland, Russia (Karelia, Leningrad, Murmansk, Yakutia), Sweden, UK; Nearctic: USA (Alaska).

\section{Rhaphium glaciale (Ringdahl, 1920)}

MATERIAL. Meynypilgyno, $62^{\circ} 37.19^{\prime} \mathrm{N}, 176^{\circ} 50.72^{\prime}$ E, lake, 2 vii 2016, P. Tomkovich, $1 \widehat{\partial}^{\jmath}$ (ZMUM).

DISTRIBUTION. Type locality: Sweden: Lappland, Hochgebirge bei Tornetrask. Palaearctic: Finland, Norway, Russia (Murmansk, Baikal, Khantia-Mansia, Krasnoyarsk, Magadan, Yakutia), Sweden; Nearctic: "North America".

\section{Rhaphium nigrum (Van Duzee, 1923)}

MATERIAL. Krasnoarmeiskii, Chaunskii District, 10 vii 1963, Gorodkov, $10^{\lambda}$ (ZIN).

DISTRIBUTION. Type locality: USA: Alaska, Katmai; Savonoski, Naknek Lake. Nearctic: USA (Alaska). New for the Palaearctic Region.

\section{Rhaphium patellitarse (Becker, 1900)}

MATERIAL. 55 km N Egvekinot along road, 29 vii 1963, Gorodkov (ZIN), 201; Iultin, 21 vii 1963, Gorodkov (ZIN), 1 ^.

DISTRIBUTION. Type locality: Russia: Khantaika [Taimyr]. Palaearctic: Russia (Altai Rep., Buryatia, Taimyria, Yakutia, Yamalia).

\section{Scellus gallicanus Becker, 1909}

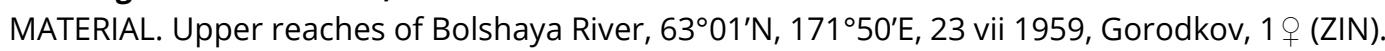

DISTRIBUTION. Type locality: France: Lautaret. Palaearctic: China, France, Mongolia, Russia (Altai Rep., Murmansk, Sayan Mnts., Irkutsk, Yakutia).

\section{Scellus spinimanus (Zetterstedt, 1843)}

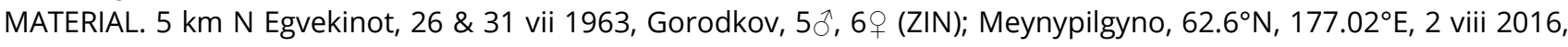
26 vii 2017, P. Tomkovich, 4ð, 4 ㅇ (ZMUM).

DISTRIBUTION. Type locality: Scandinavia. Palaearctic: Austria, Finland, Mongolia, Norway, Russia (Crimea, N Ural, Yakutia), Sweden; Nearctic: Canada (Newfoundland, Manitoba, Northwest Terr., Yukon), USA (Alaska).

\section{Sympycnus gorodkovi Negrobov, Barkalov, Selivanova et Grichanov, 2016}

REFERENCES. Negrobov et al., 2016a: 331 (Bolshaya River upper reaches).

DISTRIBUTION. Type locality: Russia: Primorye, Sikhote-Alin Biosphere Reserve, $57 \mathrm{~km} \mathrm{~W}$ Terney, left bank of Serebryanka River. Palaearctic: Russia (Blagoveshchensk, Chukotka, Khabarovsk, Magadan, Vladivostok).

\section{Conclusion}

As a result of our study, a new material of Dolichopodidae is found and identified, and a revised check-list of Chukchi species was compiled. The present research gives new records, including 26 species found for the first time in Chukotka and 3 species recorded for the first time in the Palaeartic Region. In total, 8 genera and 36 species are listed above that apparently makes up less than $50 \%$ of actual Dolichopodidae fauna of the Okrug. At present, 17 Chukchi dolichopodids (47\%) are known in the Nearctic Region.

\section{Acknowledgments}

I am grateful to Drs. E.P. Nartshuk and I.V. Shamshev (ZIN), N.E. Vikhrev and A.L. Ozerov (ZMUM) for their kindness in providing specimens for this study. The work is performed within the Program for Basic Scientific Researches in the 
Grichanov, I.Ya. An annotated checklist of Dolichopodidae of Chukotka.... Acta Biologica Sibirica, 2018, 4(2), 25-31

Russian Federation for the Long-Term Period (2013-2020) approved by Order No. 2538-r of the Government of the Russian Federation dated 27 December 2012.

\section{References}

Becker, T. (1917). Dipterologische Studien. Dolichopodidae. A. Paläarktische Region. Nova Acta Academiae Caesareae Leopodinisch-Carolinae Germanicae Naturae Curiosorum, 102(2), 113-361.

Grichanov, I.Ya. (2012a). A new peculiar species of Chrysotus from the Far East of Russia (Dolichopodidae, Diptera). Amurian zoological journal, 4(4), 333-335.

Grichanov, I.Ya. (2012b). A new species of Dolichopus Latreille, 1796 from Turkey (Diptera: Dolichopodidae). Caucasian Entomological Bulletin, 8(2), 316-318.

Grichanov, I.Ya. (2014). Alphabetic list of generic and specific names of predatory flies of the epifamily Dolichopodoidae (Diptera). St.Petersburg: VIZR, 1-544. (Plant Protection News Supplements 14).

Grichanov, I.Ya. (2017). Alphabetic list of generic and specific names of predatory flies of the epifamily Dolichopodoidae (Diptera). Second edition. St.Petersburg: VIZR, 1-563. (Plant Protection News Supplements 23).

Grichanov, I.Ya., Khrulyova, O.A. (2018). Fauna and ecology of Dolichopodidae (Diptera) from Wrangel Island Nature Reserve (Chukotka AD, Russia). Nature Conservation Research, 3(3), 23-31.

Gruzdev, A.R., Ovsyanikov, N.G., Menyushina, I.E., Khruleva, O.A., Novichkova, A., Berezyuk, L.M., Volobueva, E.A. (2014). Cadastral information on the state Nature Reserve "Wrangel Island" (for 2010-2014). Pevek: Ministerstvo prirodnykh resursov i ekologii Rossiiskoi Federatsii; FGBU «Gosudarstvennyi zapovednik “Ostrov Vrangelya”», 1-55. (In Russian)..

Hurley, R.L. (1985). Orthogenya Dolichopodidae: Hydrophorinae. In: Griffiths, G.C.D. (Ed.). Flies of the Nearctic region, 6(1), 1-112.

Maslova, O.O., Negrobov, O.P. (2015). New species of Chrysotus Meigen, 1824 (Diptera: Dolichopodidae) from Sakhalin and Kuril Islands (Russia). Caucasian Entomological Bulletin, 11(1), 201-203. (In Russian).

Maslova, O.O., Negrobov, O.P., Selivanova, O.V. (2011). Fauna of Hydrophorus alpinus Wahlberg, 1844 (Dolichopodidae, Diptera) of Russia. In: Negrobov, O.P. (Ed.). Modern Problems of Entomology. Voronezh: VSU, 93-94.

Negrobov, O.P. (1977-1979). Dolichopodidae, Unterfamilie Hydrophorinae, Unterfamilie Rhaphiinae. In: Lindner, E. (Ed.). Die Fliegen der Palaearktischen Region, Stuttgart, IV, 29, 316 (1977), 319 (1978), 321, 322 (1979), $354-530$.

Negrobov, O.P., Barkalov, A.V., Selivanova, O.V. (2012). Rhaphium Meigen (Diptera, Dolichopodidae) from the Taimyr Peninsula (Russia), with description of a new species. Zootaxa, 3548, 75-87.

Negrobov, O.P., Barkalov, A.V. \& Selivanova, O.V. (2014). Two new Chrysotus Meigen (Diptera, Dolichopodidae) from Siberia, with a key to the Siberian species. Zootaxa, 3815(3), 409416. DOI:10.11646/zootaxa.3964.3.8.

Negrobov, O.P., Barkalov, A.V., Selivanova, O.V., Grichanov, I.Ya. (2016a). Two new species of the genus Sympycnus (Diptera, Dolichopodidae) from Siberia and Russian Far East. Euroasian Entomological Journal, 15(4), 330-334. (In Russian).

Negrobov, O.P., Chalaya, O.N. (1991). Dolichopodidae (Diptera) of North-Eastern part of USSR. In: Entomological studies in the North-East of the USSR. Vladivostok: DVO AN SSSR, 66-80. (In Russian).

Negrobov, O.P., Grichanov, I.Ya. (2013). On the distribution of Dolichopus humilis Van Duzee in the Palaearctic Region and a new synonym (Diptera: Dolichopodidae: Dolichopus latipennis species group), Zootaxa, 3599(5), 499-500.

Negrobov, O.P., Maslova, O.O. (1995). Revision of Palaearctic species of genus Chrysotus Mg. (Diptera, Dolichopodidae), II. Entomologicheskoe obozrenie, 74(2), 456-466. (In Russian).

Negrobov, O.P., Maslova, O.O., Fursov, V.N. (2015). New Data on the Genus Chrysotus Meigen, 1824 (Diptera: Dolichopodidae) from Japan and Russia. Far Eastern Entomologist, 293, 10-15.

Negrobov, O.P., Mokeeva, S.Yu., Maslova, O.O., Selivanova, O.V. (2000). To the study of the family Dolichopodidae (Diptera) in the Far East. In: Biodiversity and dynamics of ecosystems in North Eurasia, Novosibirsk, 3(1), 84-86.

Negrobov, O.P., Selivanova, O.V. Maslova, O.O. (2016b). New Species of Chrysotus Meigen, 1824 (Diptera, Dolichopodidae) from Primorskii Territory. Entomological Review, 96(6), 810-818.

Negrobov, O.P., Selivanova, O.V., Maslova, O.O., Chursina, M.A. (2013). Check-list of predatory flies of the family Dolichopodidae (Diptera) in the fauna of Russia. In: Grichanov, I.Ya., Negrobov, O.P. (Eds.). Fauna and taxonomy of Dolichopodidae (Diptera). Collection of papers. St.Petersburg: VIZR, 47-93. (Plant Protection News Supplements).

\section{Citation:}

Grichanov, I.Ya. (2018). An annotated checklist of Dolichopodidae (Diptera) of Chukotka (Russia) with new records. Acta Biologica Sibirica, 4 (2), 25-31.

Submitted: 11.02 .2018 . Accepted: 15.04 .2018

cross ref http://dx.doi.org/10.14258/abs.v4i2.4120

(c) 2018 by the authors. Submitted for possible open access publication under the terms and conditions of the Creative Commons Attribution (CC BY) license (http://creativecommons.org/licenses/by/4.0/). 